\title{
Generation of Electricity using NiTinol
}

\author{
S K Haaris ${ }^{1}$ \\ Metallurgical Engineering \\ Jawaharlal Nehru Technological University \\ Hyderabad
}

\author{
G Madhu Murali Siran2 2 \\ Electrical Electronic Engineering \\ Jawaharlal Nehru Technological University \\ Hyderabad
}

\author{
D Lokesh ${ }^{3}$ \\ Metallurgical Engineering \\ Jawaharlal Nehru Technological University \\ Hyderbad
}

\begin{abstract}
This paper can be a revolution towards ecofriendly methods in both automobile and electric generation departments (industries). In this day to day life and humankind is not imaginable without electricity, moreover Electricity consumption is increasing due to new technological innovations across the world. Ironically there are many places still far away from the basic electricity needs in Indian villages. Among the different kinds of smart materials one of the most promising materials are the shape memory alloys. This paper is proposing a viable project with NiTinol material. Last two decades witnessed a tremendous change in usage of NiTinol such as it is used in stent in heart operations. NiTinol (Nickel Titanium-Naval Ordanance Laboratory) is also known as nickel titanium alloy. A thermally related shape memory property of certain nickel titanium was first observed in research at naval ordnance laboratory in the year 1959.Since that time the parallel programs in material studies, thermodynamic analysis prototypes concepts in the design of $\mathrm{Ni}$ - $\mathrm{Ti}$ heat engine have been initiated at LBL principally under a grand from national science foundation. The NiTinol crystal structure behaves in different manner when the temperature is varied, in comparison with that of other alloys. It has the special property that even if the shape is distracted by external force it can be brought into its original shape and it reacts in the same manner even when temperature is varied. This project basically converts heat energy to mechanical energy and then to electrical energy. It is affordable and also does not damage the environment. Titanium is available in large scale as it is the 9th most abundant material in earth's crust. This research can be a milestone towards revolution of automobile industries for the conservation of fuel. This paper will deal and explore with practical aspects of the use of shape memory alloy material and their applications to energy conservation.
\end{abstract}

Keywords:- NiTinol, shape memory effect, shape recovery, wire loop, energy conversion, temperature profile, heat transfer, polymer structure.

\section{INTRODUCTION}

NiTinol belongs to a class of materials called Shape Memory Alloys (SMA). SMA's have interesting mechanical properties. Flexinol which is a trade name for NiTinol actuator wire, has been trained to contract when heated, which is the opposite of what standard metals do when heated which is expand. Not only does this alloy contract, but does so with a 100 times greater thermal movement (expansion-contraction) than standard metals.
Another interesting property of SMA's is called the Shaped Memory Effect (SME). The alloy can be made to remember a particular shape. Once a shape has been remembered, the alloy may be bent out of shape. Then returned to its original shape by heating the alloy about it transition temperature.

\section{HISTORY:}

Although scientists have known about and experimented with SMA's since 1932, it wasn't until 1962 that the materials came out of the laboratory. William Beuhler, working at the US Naval labs, discovered the SMA effect in an alloy of nickel and titanium. At the time the scientific team was trying to develop a heat and corrosive resistant alloy. In the process of creating a corrosive resistant alloy, they created a Shape Memory Alloy (SMA) made of 55\% nickel and $45 \%$ titanium. This new material was relatively inexpensive and much safer (non-toxic) than previous SMA's.

The team named their new alloy NiTinol (pronounced night-in-all). The name represents its elemental components and place of origin. The "Ni" and "Ti" are the atomic symbols for nickel and titanium. The "NOL" stands for the Naval Ordinance Laboratory where it was discovered.

Although the mixture of nickel to titanium in NiTinol is about equal, the smallest change in the ratio of the two compounds has a dramatic effect on the transition temperature of the resulting alloy. For instances, a $1 \%$ difference in the ratio varies the transition temperature from -100 to +100 C. Every company manufacturing NiTinol products today must hold the ratio of the components to a precise level to insure a stable and repeatable transition temperature. The NiTinol alloy we are experimenting with has a transition temperature of $70 \mathrm{C}$ (158F).

\section{HOW IT WORKS:}

The properties of NiTinol depend upon its dynamic heat sensitive crystalline structure. When NiTinol is deformed in the martensite phase, the crystalline structure is not damaged. Instead the crystal structure transforms moving in a singular crystalline direction. When heated the material returns to its "remembered" lest stress austenite structure. 
In normal metals, deformation causes the dislocations of the molecular structure into new crystal positions. There is no "memory" in the crystal of where the atoms were before they moved.

The physical movement of NiTinol is attributed to internal molecular restructuring. And because movement is generated on a molecular level, it is quite strong.

\section{PHYSICAL PROPERTIES:}

Tensile Strength --- 200,000 psi

Melting Point --- 1,250C (2,282 F)

Resistance --- $1.25 \mathrm{ohms}$ per inch (.006 wire)

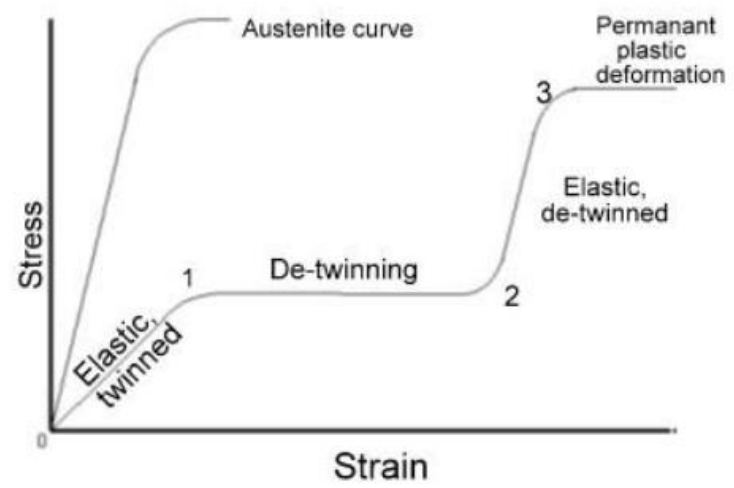

STRESS- STRAIN RELATIONSHIP

A quick overview of NiTinol phases are: NiTinol at room temperature is in its martensitic phase. When the alloy is bent, crystalline structure deforms, creating internal stress. Heating the alloy above its transition temperature (Austenite phase), the crystalline structure tries to relieve any stress in its crystalline structure by returning to its original "remembered" shape. This creates the thermal movement of the SMA.

\section{SUPER ELASTICITY:}

Super elasticity is a unique property of SMA. If the SMA is deformed at a temperature slightly above its transition temperature, it springs right back into shape. This springy property is called super elasticity. Manufacturers have capitalized on this property finding useful medical and dental applications.

For instance, low temperature NiTinol wire is used as orthodontic arch wires in braces. The NiTinol wire provides a low constant force at human body temperature used to straighten teeth while reducing the need for wire retightening. The transition temperature of these wires are made so that they generate force at the temperature of the human mouth (about 37C (98.6F))

\section{APPLICATIONS:}

- This alloy is very useful in dentistry, especially in orthodontics for wires and brackets that connect the teeth. Sure Smile (a type of braces) is an example of its orthodontic application.

- It is also used in endodontic mainly during root canals for cleaning and shaping the root canals.

- In colorectal surgery, it is used in various devices for the purpose of reconnecting the intestine after the pathology is removed.

- NiTinol stents are another significant application of this metal in medicines.

- Its biocompatible properties make useful in orthopedic implants.

- NiTinol wires can be used for marking and locating breast tumors.

- $\quad$ The use of NiTinol tubing for various medical purposes is increasing in popularity.

- This material is used in temperature controls. Its shape changing properties can be used for activating a variable resistor or a switch for controlling the temperature.

- It is used as microphone boom or a retractable antenna in cell phone technology for its mechanical and flexible memory nature.

- NiTinol spring is used in various industries for the purpose of utilizing the super elastic properties of this metal.

\section{Experimental setup:}

$\rightarrow$ the engine generates power by using NiTinol wire loop. The loop made of NiTinol runs in between the two pulleys. Hot water on hot side and cool ambient air on cool side is used by this device in order to perform action.

$\rightarrow$ The smaller wheel of engine is partially dipped in a hot liquid. Memory given to the NiTinol loop wire in engine is 'straight' shape. Whenever a part of loop gets in contact with a hot liquid, it gets heated above its transition temperature and tries to straighten out.

$\rightarrow$ NiTinol wire is straight and cool. As the wire travels from position 1 to position 2, it gets bent around the small glass pulley and comes in contact with hot liquid. As the wire moves from position 2 to position 3, it comes in contact with hot water and thus gets heated above its transition temperature due to which it tries to straighten out. As the wire tries to straighten out, it takes form the form shown by dotted line.

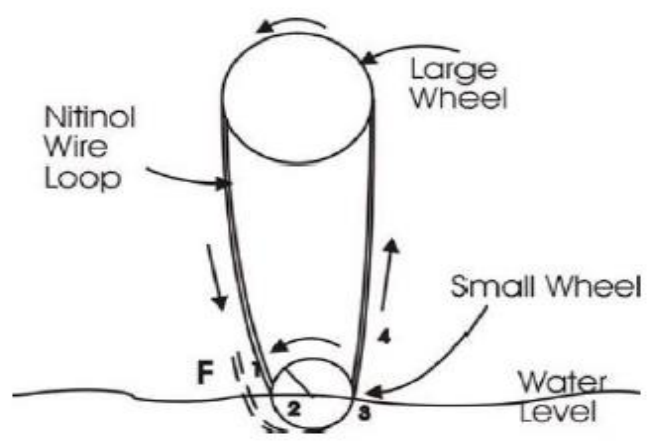

$\rightarrow$ As the wire moves from position 3 to 4 , it comes in contact with ambient air due to which its temperature 
decreases and, thus, austenite phase starts converting to martensite phase. As the wire moves from position 1 to position 4 , it travels over the bigger pulley and a sufficient long time is available for the wire to cool below its transition temperature; the wire get ready for another cycle.

\section{Experiments performed:}

Water is heated to 90 degrees Celsius with help of conventional heating process and then the heating source is removed. Water is then poured in a flask and NiTinol engine is placed over the flask. The temperature on cool side is room temperate which was found to be 32 degrees Celsius during the experiment.

As explained in working principle in the above section, the engine starts rotating. Speed of engine is measured with the help of laser tachometer. Laser tachometer used for measuring speed of NiTinol engine in RPM. As the time passes, temperature of water bath goes on decreasing due to heat loss to surrounding. Variation in speed of the engine is measured with the help of laser tachometer.

RESULTS AND CONCLUSIONS:

\begin{tabular}{|l|c|l|}
\hline S.NO: & TEMPERATURE & RPM \\
\hline 1 & 90 & 180 \\
\hline 2 & 87 & 180 \\
\hline 3 & 84 & 180 \\
\hline 4 & 81 & 169 \\
\hline 5 & 78 & 165 \\
\hline 6 & 75 & 155 \\
\hline 7 & 72 & 130 \\
\hline 8 & 69 & 115 \\
\hline 9 & 66 & 107 \\
\hline
\end{tabular}

THE ABOVE TABLE SHOWS THE NO. OF ROTATIONS PER MINUTE AT DIFFERENT TEMPERATURE OF NITINOL WIRE

This experiment was conducted on different bulbs of two different voltages. When the experiment was conducted by 2.5 watts LED bulb the bulb glows till the temperature reaches to 72 degrees and the experiment was also conducted on 5 watts LED bulb the bulb glows till the temperature reaches to 84 degrees.

\section{SUMMARY:}

Thermal parameters influencing the speed of the $\mathrm{Ni}-\mathrm{Ti}$ engine are determined. Results show that the temperature of bath plays a major role on power output.

\section{REFERENCES:}

[1] Liu, Y, humbleeck, J.V., stalmans, r., delaey, L., 1997. Some aspects of the properties of the NiTi shape memory alloy. J. alloys compd. 247,115-121

[2] Shape memory materials by Arun D I, Chakravarthy P, Arockia Kumar R, Santhosh B.

[3] Cederström, J.; Van Humbeeck, J. (February 1995). "Relationship Between Shape Memory Material Properties and Applications". Le Journal de Physique IV. .

[4] Filip, Peter; Mazanec, Karel (May 1995). "Influence of work hardening and heat treatment on the substructure and deformation behaviour of TiNi shape memory alloys". Scripta Metallurgica et Materialia.

[5] Huang, S; Leary, Martin; Attalla, Tamer; Probst, K; Subic, A (2012). "Optimisation of Ni-Ti shape memory alloy response time by transient heat transfer analysis". Materials \& Design. 